ISSN 0103-8478

\title{
Determinação da temperatura base para o desenvolvimento embrionário da mosca-da-bicheira
}

\author{
Assessment of the threshold temperature for the embryonic development of the screwworm fly
}

\author{
Thiago Mastrangelo ${ }^{\mathrm{I}}$ Fernando Bezerra $^{\mathrm{II}}$ Thiago Fernandes ${ }^{\mathrm{III}}$
}

RESUMO

Após sucessivas campanhas de erradicação da mosca-da-bicheira, Cochliomyia hominivorax (Coquerel), através da utilização de insetos estéreis, sua distribuição geográfica atual abrange apenas áreas do Caribe e dos países da América do Sul, com exceção do Chile. Para o planejamento de novos programas de controle nos países do MERCOSUL, deve-se contar com o máximo de informações possíveis relacionadas à ecologia desse parasita, como suas exigências térmicas. O objetivo deste estudo foi determinar a constante térmica $(K)$, as temperaturas base $\left(T_{b}\right)$, ótima $\left(T_{0}\right)$ e a influência de temperaturas entre 15 e $35^{\circ} \mathrm{C}$ sobre a duração do período embrionário de C. hominivorax. Depois de separados das massas, os ovos foram inoculados sobre papel de filtro umedecido em placas de Petri, as quais foram colocadas em câmaras climatizadas do tipo B.O.D. Considerando o menor período de desenvolvimento e máxima viabilidade de ovos, a temperatura ótima foi estimada em $35^{\circ} \mathrm{C}$. A temperatura base foi estimada em $13,1^{\circ} \mathrm{C}$, com uma constante térmica de 9,17GD.

Palavras-chave: miíase, constante térmica, temperatura base, Cochliomyia hominivorax.

\section{ABSTRACT}

After successful eradication campaigns against the screwworm fly, Cochliomyia hominivorax (Coquerel), through the release of sterile insects, its current geographical distribution comprehends only some Caribbean islands and South American countries, except Chile. Before planning new control programs in MERCOSUR countries, information related to the ecology of this parasite, as its thermal requirements, are necessary. This research aimed to assess the thermal constant $(K)$, the threshold $\left(T_{b}\right)$ and optimal $\left(T_{o}\right)$ temperatures, as the influence of temperature between 15 and $35^{\circ} \mathrm{C}$ over the length of the embryonic period of $\boldsymbol{C}$. hominivorax. Eggs were separated from the masses and inoculated on moistened filter paper in Petri dishes, which were held in acclimatized chambers. Considering the shortest development period and the highest egg hatch values, the optimal temperature was $35^{\circ} \mathrm{C}$. The threshold temperature was $13.1^{\circ} \mathrm{C}$ and the estimated thermal constant was 9.17 degrees-days.

Key words: myiasis, thermal constant, threshold temperature, Cochliomyia hominivorax.

\section{INTRODUÇÃO}

A mosca Cochliomyia hominivorax (Coquerel) é um parasita obrigatório de animais de sangue quente e provoca mí́ase primária em feridas pré-existentes. Sua distribuição abrangia originalmente o sudoeste dos EUA, passando pelo cinturão tropical da Flórida, até as Américas Central e do Sul (HALL \& WALL, 1995; IAEA, 2008). Após sucessivas campanhas de erradicação através da utilização de insetos estéreis a partir da década de 1960, C. hominivorax está presente hoje apenas em algumas áreas da região do Caribe (Cuba, República Dominicana, Haiti, Jamaica, Trinidad e Tobago) e nos países da América do Sul, com exceção do Chile (IAEA, 2008).

Em 2006, a Comisión México-Americana para la Erradicación del Gusano Barrenador del Ganado (COMEXA) realizou um projeto-piloto na fronteira entre Uruguai e Brasil, onde os prejuízos

'Universidade Estadual de Campinas (UNICAMP), 13083-875, Campinas, SP, Brasil. E-mail: mastrangelo@cbmeg.unicamp.br. Autor para correspondência.

"Universidade Federal do Vale do São Francisco (UNIVASF), Petrolina, PE, Brasil.

"'Universidade Metodista de Piracicaba (UNIMEP), Piracicaba, SP, Brasil. 
causados na pecuária por essa mosca somavam US\$ 150 mil/ano. No final das 13 semanas de liberação de moscas estéreis, o projeto demonstrou resultados positivos de controle (PONTES et al., 2009). Para o planejamento de novos programas de supressão ou erradicação de C. hominivorax para os países do MERCOSUL (Mercado Comum do Sul), devese contar com o máximo de informações possíveis relacionadas à ecologia e dinâmica das populações naturais dessa praga .

A temperatura é um dos fatores ecológicos mais importantes para o desenvolvimento e o comportamento dos insetos de modo geral. Cada espécie apresenta um requisito térmico próprio, determinando a maior ou menor adequação do ambiente para o seu crescimento populacional (SALVADORI \& PARRA, 1990).

Os estudos de exigências térmicas, em condições controladas de laboratório, fornecem subsídios importantes para o desenvolvimento de modelos em graus-dia, os quais são muito utilizados em ecologia e manejo de pragas, sendo também importantes para a previsão de ocorrência de pragas e inimigos naturais (HENCE et al., 2007) e para o zoneamento ecológico de pragas (MILANEZ \& PARRA, 2000).

A dinâmica populacional de $C$. hominivorax é influenciada diretamente pela disponibilidade de hospedeiros e pelas variações dos fatores climáticos (ADAMS 1979; KRAFSUR \& LINDQUIST, 1996; MASTRANGELO \& WELCH, 2012). Na literatura, entretanto, poucos autores investigaram as exigências térmicas relacionadas à oviposição de C. hominivorax. Segundo ADAMS (1979), variações de temperatura dentro da faixa ótima $\left(21,8^{\circ}\right.$ a $\left.37,8^{\circ} \mathrm{C}\right)$ provocam poucas mudanças na maturação dos ovos pelas fêmeas, mas, como exemplo, um aumento de $5,5^{\circ} \mathrm{C}$ dentro de faixas subótimas (de $37,8^{\circ}$ a $43,3^{\circ} \mathrm{C}$, por exemplo) pode provocar uma redução da longevidade dos adultos de até 57,7\%. Segundo o mesmo autor, a maior taxa de maturação dos ovos foi verificada a $32,2^{\circ} \mathrm{C}$, e temperaturas acima desta estressariam o inseto, aumentando o tempo necessário para completar a prévitelogênese e a vitelogênese (ADAMS, 1979).

$$
\text { KRAFSUR \& GARCIA (1978) }
$$

demonstraram que o momento em que $90 \%$ de uma população de $C$. hominivorax oviposita pela $1^{\text {a }}$ vez pode ser estimado por uma equação linear em função da temperatura $(D-1=98.04 /(T-8.41)$, em que $D$ é o tempo de desenvolvimento, em dias, e $T$ é temperatura). Outras observações de laboratório e campo demonstraram que o dia modal para a $1^{\underline{a}}$ oviposição, a temperaturas $\geq 27^{\circ} \mathrm{C}$, ocorre no $5^{\circ}$ dia de idade da mosca adulta, com as oviposições subsequentes podendo ocorrer a cada três dias (CRYSTAL, 1967; HIGHTOWER et al., 1972; MANGAN \& THOMAS, 1989; THOMAS \& CHEN, 1990). DAVIS et al. (1968) verificaram que, em temperaturas inferiores a $20^{\circ} \mathrm{C}$, as fêmeas reduzem significativamente a oviposição, o que poderia atuar como fator limitante ao crescimento populacional do parasita.

No entanto, considerando-se a influência da temperatura sobre a eclosão das larvas de massas de ovos de C. hominivorax, a relação ainda não está clara na literatura. Poucos estudos investigaram essa influência especificamente, não havendo padronização de metodologias (incluindo as faixas de temperatura) e sendo a maioria apenas observações de campo.

Dois dos parâmetros mais comumente empregados para avaliar essa influência térmica são a temperatura base $(\mathrm{Tb})$ e a temperatura ótima (To). A primeira se refere a uma temperatura a partir da qual é possível ocorrer o desenvolvimento embrionário, enquanto a segunda é definida como sendo aquela temperatura em que há a máxima viabilidade (eclosão de larvas) num curto período de tempo. As necessidades térmicas dos insetos também podem ser avaliadas pela constante térmica $(\mathrm{K})$, a qual é usada tradicionalmente em estudos de previsão de crescimento de plantas. Uma vez que os insetos são heterotérmicos, essa constante térmica também pode ser aplicada ao desenvolvimento dos insetos (HADDAD \& PARRA, 1984).

Este estudo, portanto, teve como objetivo a determinação da influência de temperaturas entre 15 e $35^{\circ} \mathrm{C}$ sobre a duração do período embrionário de $\boldsymbol{C}$. hominivorax, além de determinar a constante térmica ( $\mathrm{K}$, em graus-dias) e as temperaturas base $\left(\mathrm{T}_{\mathrm{b}}\right)$ e ótima $\left(\mathrm{T}_{\mathrm{o}}\right)$, em condições controladas de laboratório.

\section{MATERIAL E MÉTODOS}

Os ovos foram obtidos de 30 fêmeas com 7 dias de idade da colônia de $\boldsymbol{C}$. hominivorax mantida no Centro de Energia Nuclear na Agricultura (CENA/ USP). Para maximizar a oviposição das moscas, a temperatura da sala devia estar maior ou igual a $26^{\circ} \mathrm{C}$ e a umidade relativa do ar maior ou igual a $80 \%$. 
Como substrato de oviposição, foi utilizada uma mistura de resto de dieta larval, coágulo e sangue bovino citratado (MASTRANGELO \& WELCH, 2012). Essa mistura era colocada em tampa plástica de cor preta, coberta com recortes de papel de filtro e aquecida até $35^{\circ} \mathrm{C}$ em estufa, depois era oferecida às moscas. Depois de 3-4h de exposição na gaiola com as 30 fêmeas, esse substrato de oviposição era retirado do interior da gaiola e as massas de ovos eram então coletadas. A eclosão de larvas foi avaliada segundo BERKEBILE \& SKODA (2002), sendo a massa de ovos agitada em solução de $\mathrm{NaOH}$ a $1,2 \%$ por $5 \mathrm{~min}$, e os ovos enxaguados e peneirados.

Depois de separados das massas, 100 ovos foram inoculados sobre papel de filtro umedecido em placas de Petri (10 cm de diâmetro), as quais foram vedadas na lateral com parafilme Magipack $^{\circledR}$. O papel foi umedecido diariamente $(2 \mathrm{~mL})$, de forma a evitar a desidratação dos ovos. As placas ficaram em câmaras climatizadas do tipo B.O.D. (12h de fotofase e $65 \pm 2 \%$ de UR), reguladas conforme os tratamentos representados pelas seguintes temperaturas: 15; 19,5; 25 e $35^{\circ} \mathrm{C}$.

A avaliação foi realizada diariamente por cinco dias. Cada uma das placas foi considerada uma parcela experimental, havendo três repetições/ tratamento. A determinação da temperatura base e da constante térmica foi feita através do método da hipérbole (HADDAD \& PARRA, 1984). As médias de duração do período embrionário e viabilidade de ovos foram comparadas pelo teste de Tukey $(\alpha=0,05)$. As análises foram realizadas através do programa estatístico SAS 9.1 (SAS Institute, 2003).

\section{RESULTADOS E DISCUSSÃO}

O período de incubação e a viabilidade de ovos variaram em função das temperaturas testadas, caracterizando uma relação inversamente proporcional entre a duração do desenvolvimento embrionário e a temperatura (Figura 1), dentro de uma faixa de 15 a $35^{\circ} \mathrm{C}$. As menores viabilidades (Tabela 1) foram encontradas para a temperatura de $15^{\circ} \mathrm{C}$, provavelmente em razão da menor atividade metabólica dos ovos. A faixa térmica entre 25 e $35^{\circ} \mathrm{C}$ proporcionou maior viabilidade de ovos $\left(F=134,1 ; P<10^{-3}\right)$. Considerando um menor período de desenvolvimento e máxima viabilidade de ovos, a temperatura ótima, dentre as testadas, seria de $35^{\circ} \mathrm{C}$ (Tabela 1).

Com base na equação de regressão linear (Tabela 2), a temperatura base foi estimada em $13,1^{\circ} \mathrm{C}$, com uma constante térmica de $9,17 \mathrm{GD}$. Essas estimativas estão de acordo com a literatura. Segundo HONÉK (1996), a maioria das espécies de insetos que vivem em regiões tropicais possui uma $T_{b}$ média $\left(13,7^{\circ} \mathrm{C}\right)$ maior do que aqueles que vivem em regiões de clima subtropical $\left(10,5^{\circ} \mathrm{C}\right)$ ou temperado $\left(7,9^{\circ} \mathrm{C}\right)$. Vale também ressaltar que o limiar térmico inferior pode variar para uma mesma espécie, em função do local e clima em que se encontra a população, estado fisiológico do inseto, estágio de desenvolvimento, fotoperíodo e qualidade nutricional das fontes alimentares (BERGANT \& TRDAN, 2006).

A constante térmica estimada para $C$. hominivorax $(9,17 \mathrm{GD})$ foi pequena. Como exemplo, a $35^{\circ} \mathrm{C}$, de acordo com o valor estimado, a completa

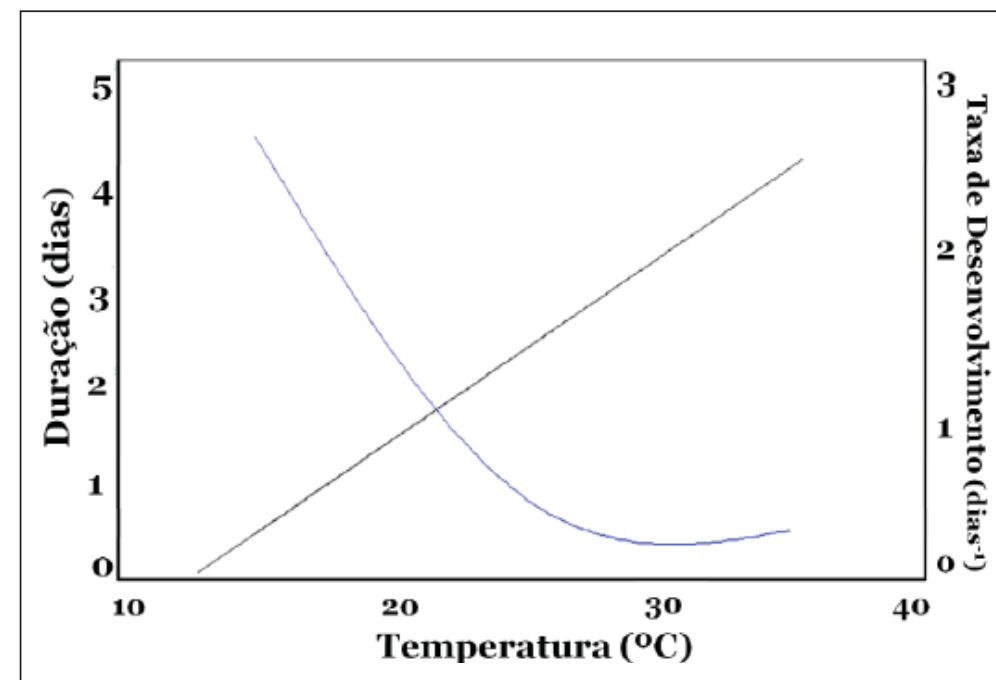

Figura 1 - Relação entre a temperatura com a duração (hipérbole) e a taxa de desenvolvimento (reta) para a fase de ovo de Cochliomyia hominivorax em condições de laboratório. 
Tabela 1 - Médias ( \pm erro padrão) da duração do desenvolvimento embrionário (dias) e viabilidade de ovos (\%) de Cochliomyia hominivorax em diferentes temperaturas de incubação (UR $=60 \% \pm 10 \%$, fotofase $=12 \mathrm{~h}$ ).

\begin{tabular}{llc}
\hline Temperatura $\left({ }^{\circ} \mathrm{C}\right)$ & Duração (dias) & Viabilidade $(\%)$ \\
\hline 15 & $3 \pm 0,6 \mathrm{a}^{\mathrm{a}}$ & $30,3 \pm 3,9 \mathrm{c}^{\mathrm{b}}$ \\
19,5 & $1,5 \pm 0,05 \mathrm{~b}$ & $70,4 \pm 4,1 \mathrm{~b}$ \\
25 & $0,9 \pm 0,06 \mathrm{bc}$ & $82,3 \pm 3,0 \mathrm{ab}$ \\
35 & $0,4 \pm 0,05 \mathrm{c}$ & $91,9 \pm 1,5 \mathrm{a}$ \\
C.V. & $16,8 \%$ & $8,3 \%$ \\
F & 30,5 & 134,1 \\
$\mathrm{P}$ & $<10^{-3}$ & $<10^{-3}$ \\
\hline
\end{tabular}

${ }^{a}$ Médias originais nesta tabela. Os dados de duração do desenvolvimento embrionário foram transformados por " $\log _{10}(\mathrm{x}+\mathrm{k})$ " antes das análises.

${ }^{\mathrm{b}}$ Médias na mesma coluna, seguidas da mesma letra, não diferem significativamente entre si pelo teste de Tukey $(\mathrm{P}>0,05)$.

eclosão das larvas se daria em até $10 \mathrm{~h}$, o que já foi observado em laboratório e hospedeiros (GUEVARA, 1986; SPRADBERY, 1994).

A partir de $13^{\circ} \mathrm{C}$, portanto, o aumento populacional de $\boldsymbol{C}$. hominivorax passaria a depender mais da qualidade e abundância de hospedeiros (PARMAN, 1945; DEONIER, 1946; HIGHTOWER, 1963; JONES et al., 1976; MASTRANGELO \& WELCH, 2012).

PARMAN (1945), baseado em dados de campo, observou que as populações naturais de C. hominivorax nos EUA diminuíam quando as temperaturas mensais eram menores do que $15,6^{\circ} \mathrm{C}$ e não sobreviviam a invernos com temperaturas médias menores que $9,5-10,2^{\circ} \mathrm{C}$. Estudos posteriores (LINDQUIST \& BARRETT, 1945; PARISH, 1945; BRODY \& ROGERS, 1945; DEONIER, 1945; FLITTERS \& BENSHOTTER, 1968) também corroboraram essa faixa de $9-10^{\circ} \mathrm{C}$ por três meses como limitante à sobrevivência de $\boldsymbol{C}$. hominivorax.

Um manejo mais cuidadoso do rebanho e animais domésticos nos meses mais frios poderia contribuir significativamente na diminuição da população local (PARMAN, 1945; DEONIER, 1945). Localidades da América do Sul que possuem longos períodos com temperaturas menores que $9-13^{\circ} \mathrm{C}$ poderiam até servir como barreiras temporárias contra a expansão de populações de $\boldsymbol{C}$. hominivorax durante uma campanha de supressão, mesmo sob menor aplicação de inseticidas ou baixa quantidade de machos estéreis liberados na área de barreira temporária (IAEA, 2008).

As informações referentes ao efeito da temperatura no desenvolvimento de $\boldsymbol{C}$. hominivorax podem auxiliar não só na elaboração de futuros mapas de zoneamento ecológico desse parasita, como também são extremamente importantes para a criação das moscas em laboratório. Como exemplo, estando a temperaturas entre $19-24^{\circ} \mathrm{C}$, as massas de ovos podem aguardar até $20 \mathrm{~h}$ antes se serem adicionadas à dieta larval, sem haver grandes prejuízos à viabilidade larval (eclosão variando entre 70 e 80\%) (Tabela 1).

\section{CONCLUSÃO}

A temperatura base para o desenvolvimento embrionário de Cochliomyia hominivorax é de $13,1^{\circ} \mathrm{C}$, com uma constante térmica de 9,17GD, sendo estes dados úteis para a elaboração de futuros mapas de zoneamento ecológico desse parasita e, principalmente, para a criação das moscas em laboratório.

\section{AGRADECIMENTOS}

À Fundação de Amparo à Pesquisa do Estado de São Paulo (FAPESP), pela concessão da bolsa de Doutorado, e ao Conselho Nacional de Desenvolvimento Científico e Tecnológico (CNPq), pela concessão das bolsas de iniciação científica.

Tabela 2 - Limiar térmico inferior de desenvolvimento (Tb), constante térmica (K), taxa de desenvolvimento (1/D) expressa pela equação de regressão e coeficiente de determinação $\left(\mathrm{r}^{2}\right)$ da fase de ovo de Cochliomyia hominivorax, em condições de laboratório (UR $=$ $60 \% \pm 10 \%$; fotofase $=12 \mathrm{~h}$ )

\begin{tabular}{lllcrrrrr}
\hline Fase & $\mathrm{Tb}\left({ }^{\circ} \mathrm{C}\right)$ & $\mathrm{K}(\mathrm{GD})$ & Equação de regressão & $\mathrm{r}^{2}$ & \multicolumn{2}{c}{$\begin{array}{c}\text { Erro padrão do coeficiente } \\
\text { angular }\end{array}$} & \multicolumn{2}{c}{$\begin{array}{c}\text { Erro padrão do coeficiente } \\
\text { linear }\end{array}$} \\
\hline Ovo & 13,1 & 9,17 & $1 / \mathrm{D}=-1,429+0,109 . \mathrm{T}$ & 0,97 & 0,012 & 0,29 & 83,03 & 0,011 \\
\hline
\end{tabular}




\section{REFERÊNCIAS}

ADAMS, T.S. The reproductive physiology of the screwworm, Cochliomyia hominivorax (Diptera: Calliphoridae). II. Effect of constant temperatures on oogenesis. Journal of Medical Entomology, Lanham, v.15, p.484-487, 1979. Disponível em: <http:// esa.publisher.ingentaconnect.com/content/esa/jme/1979/00000015/ F0020005/art00011>. Acesso em: 10 de janeiro de 2013.

BERGANT, K.; TRDAN, S. How reliable are thermal constants for insect development when estimated from laboratory experiments? Entomologia Experimentalis et Applicata, Amsterdam, v. 120, p. 251-256, 2006. Disponível em: doi: 10.1111/J.15707458.2006.00433.X. Acesso em: 10 de janeiro de 2013.

BERKEBILE, D.R.; SKODA, S.R. Chemicals useful for separating egg masses of the screwworm. Southwestern Entomologist, Dallas, v.27, p.97-299, 2002. Disponível em: <http://sswe.tamu. edu/articles/PDF/SWE_V27_N3-4_P297-299.pdf $>$. Acesso em: 15 de janeiro de 2013.

BRODY, A.L.; ROGERS, E.E. Winter activity of Cochliomyia americana in the Southeastern States. Journal of Economic Entomology, College Park, v.38, n.1, p.85-89, 1945. Disponível em: <http:/www.cabdirect.org/abstracts/19461000440.html;jsessio nid=31E206EA2F40A4880DEDDF6E29489F37?freeview=true $>$. Acesso em: 10 de janeiro de 2013.

CRYSTAL, M.M. Reproductive behavior of laboratory-reared screw-worm flies (Diptera: Calliphoridae). Journal of Medical Entomology, Lanham, v.4, n.4, p.443-450, 1967. Disponível em: $<$ http://www.ncbi.nlm.nih.gov/pubmed/5623783>. Acesso em: 10 de janeiro de 2013

DAVIS, R.B.; CAMINO, M. Life cycle of the screwworm reared in outdoor cages near Veracruz City, Mexico. Journal of Economic Entomology, College Park, v.61, p.824-827, 1968 Disponível em: <http://www.ingentaconnect.com/content/esa/ jee/1968/00000061/00000003/art00064. Acesso em: 10 de janeiro de 2013 .

DEONIER, C.C. Overwintering and dispersion of Cochliomyia americana in Arizona. Journal of Economic Entomology, College Park, v.38, p.90-95, 1945. Disponível em: <http://www. cabdirect.org/abstracts/19461000441.html? freeview=true $>$. Acesso em: 10 de janeiro de 2013.

DEONIER, C.C. Population studies on Cochliomyia americana in Arizona. Journal of the Kansas Entomological Society, Manhattan, v.19, p.26-29, 1946. Disponível em: <http://www. jstor.org/stable/25081792>. Acesso em: 10 de janeiro de 2013.

FLITTERS, N.E.; BENSCHOTER, C.A. Survival of Screwworm Pupae Exposed to Simulated Winter Temperatures from Selected Sites in Texas. Annals of the Entomological Society of America v.61, p.65-67, 1968. Disponível em: < http://www. ingentaconnect.com/content/esa/aesa/1968/00000061/00000001/ art00015? crawler $=$ true $>$.

GUEVARA, J.D.E. Biologia de duas amostras brasileiras de Cochliomyia hominivorax (Diptera: Calliphoridae) submetidas ou não a irradiação gama. 1986. 49 p. Dissertação (Mestrado) Universidade Federal de Minas Gerais, Belo Horizonte, 1986.

HADDAD, M.L.; PARRA, J.R.P. Métodos para estimar os limites térmicos e a faixa ótima de desenvolvimento das diferentes fases do ciclo evolutivo de insetos. Piracicaba:FEALQ, 1984. 12 p. (Série Agricultura e Desenvolvimento).

HALL, M.; WALL, R. Myiasis of human and domestic animals. Advances in Parasitology, London, v. 35, p. 257-334, 1995. Disponível em: < http://www.ncbi.nlm.nih.gov/pubmed/7709854>.

HENCE, T.; et al. Impact of extreme temperatures on parasitoids in a climate change perspective. Annual Review of Entomology, v.52, p.107-126, 2007. DOI: 10.1146/annurev.ento.52.110405.091333.

HIGHTOWER, B.G. Nocturnal resting places of the screw-worm fly. Journal of Economic Entomology, College Park, v. 56, p. 498-500, 1963. Diponível em: <http://www.ingentaconnect.com/ content/esa/jee/1963/00000056/00000004/art00022>.

HIGHTOWER, B.G.; ET AL. Ovipositional behavior of wild-type and laboratory adapted strains of screwworm flies. Environmental Entomology, College Park, v. 1, p. 227-229, 1972. Disponível em: <http://www.ingentaconnect.com/content/ esa/envent/1972/00000001/00000002/art00023>.

HONÉK A. Geographical variation in thermal requirements for insect development. European Journal of Entomology, v. 93, p.303-312, 1996. Disponível em: <http://www.eje.cz/ pdfarticles/650/eje_093_3_303_Honek.pdf>.

IAEA. Model business plan for a sterile insect production facility. Vienna, Austria, 2008. 396 p. (Project INT/5/145 Insect Pest Control Using the Sterile Insect Technique).Disponível em: $<$ http://www-pub.iaea.org/MTCD/publications/PDF/IAEA-MBP web.pdf $>$

JONES, C.M.; et al. Screwworm flies: seasonal occurrence in central Tamaulipas, Mexico, 1973-1974. Journal of Economic Entomology, College Park, v. 69, p. 761-762, 1976. Disponível em: <http://www.ingentaconnect.com/content/esa/ jee/1976/00000069/00000006/art00019?crawler=true $>$.

KRAFSUR, E.S.; LINDQUIST, D.A. Did the sterile insect technique or weather eradicate screwworms (Diptera: Calliphoridae) from Libya? Journal of Medical Entomology, Lanham, v. 33, p. 877-887, 1996. Disponível em: <http://www. ingentaconnect.com/content/esa/jme/1996/00000033/00000006/ art00001? crawler $=$ true $>$.

KRAFSUR, E.S.; GARCIA, L. Responses of the screwworm, Cochliomyia hominivorax, to two sterile male release methods in south Texas, 1975-1976. Journal of Medical Entomology, Lanham, v.14, p.687-697, 1978. Disponível em: <http://www. researchgate.net/publication/233515135_RESPONSES_OF_ THE SCREWWORM COCHLIOMYIA HOMINIVORAX TO_TWO_STERILE_MALE_RELEASE_METHODS_IN SOUTTH_TEXAS_1975-1976? ev=sim_pub>.

LINDQUIST, A.W.; BARRETT, W.L. Overwintering of Cochliomyia americana at Uvalde, Texas. Journal of Economic Entomology, v.38, p.77-83, 1945. Disponível em: <http://www. cabdirect.org/abstracts/19461000438.html? freeview=true $>$.

MANGAN, R.L.; THOMAS, D.B. Habitat preferences and dispersal patterns in native screwworm flies (Diptera: Calliphoridae). Annals of the Entomological Society of America, Columbus, v. 82, p. 332-339, 1989.Disponível em: <http://www. ingentaconnect.com/content/esa/aesa/1989/00000082/00000003/ art00012?crawler $=$ true $>$. 
MASTRANGELO, T.; WELCH, J.B. An Overview of the Components of AW-IPM Campaigns against the New World Screwworm. Insects (2075-4450), v. 3 Issue 4, 930-955, 2012. doi: $10.3390 /$ insects 3040930

MILANEZ, J.M.; PARRA, J.R.P. Biologia e exigências térmicas de Diabrotica speciosa (Germar) (Coleoptera: Chrysomelidae) em laboratório. Anais da Sociedade Entomológica do Brasil, Londrina, v. 29, p. 23-29, 2000. Disponível em: <http://dx.doi. org/10.1590/S0301-80592000000100003>

PARISH, H.E. Overwintering of Cochliomyia americana and C. macellaria at Menard, Texas. Journal of Economic Entomology, v.38, p.83-84, 1945. Disponível em: <http://www. cabdirect.org/abstracts/19461000439.html? freeview=true>

PARMAN, D.C. Effect of weather on Cochliomyia americana and a review of methods and economic applications of the study. Journal of Economic Entomology, College Park, v. 38, p. 66-76, 1945. Disponível em: <http://www.cabdirect.org/ abstracts/19461000437.html?freeview=true>

PONTES, J.B.; et al. Projeto demonstrativo de controle e possível erradicação da mosca da bicheira. Hora Veterinária, Porto Alegre, n. 171, p. 27-30, 2009. Disponível em: <http://www.cabdirect.org/ abstracts/20093351186.html; jsessionid=123B3F94BDF3966362D 598AFD7AD6704>

SALVADORI, J.R.; PARRA, J.R.P. Efeito da temperatura na biologia e exigências térmicas de Pseudaletia sequax (Lepidoptera: Noctuidae), em dieta artificial. Pesquisa Agropecuária Brasileira, Brasília, v. 25, n. 12, p. 1693-1700, 1990. Disponível em: <http://www.bdpa.cnptia. embrapa.br/busca? $=$ =pc \&id= $824018 \&$ biblioteca $=$ vazio \&busca $=$ auto ria:\%22PARRA,\%20J.R.P.\%22\&qFacets=autoria:\%22PARRA,\%20 J.R.P.\%22\&sort $=\&$ paginacao $=t \&$ paginaAtual $=1>$

SPRADBERY, J.P. Screw-worm fly: a tale of two species. Agricultural Zoology Reviews, Bedfordshire, UK, v. 6, p. 1-61, 1994. Disponível em: <http://www.xcsconsulting.com.au/pdf/ Two_Species.pdf $>$

STATISTICAL ANALYSIS SYSTEM. SAS Version 9.1. Cary, NC: SAS Institute Inc., 2003.

THOMAS, D.B.; CHEN, A.C. Age distribution of adult female screwworms (Diptera: Calliphoridae) captured on sentinel animals in the coastal lowlands of Guatemala. Journal of Economic Entomology, College Park, v. 83, p. 1422-1429, 1990. Disponível em: <http://www.ingentaconnect.com/content/esa/ jee/1990/00000083/00000004/art00046?crawler=true> 